\title{
Towards Realistic Facial Modeling and Re-Rendering of Human Skin Aging Animation
}

\author{
Hussein Karam Hussein ${ }^{1,2}$ \\ ${ }^{1}$ Ain Shams University \\ Faculty of Science \\ Mathematics and Computer Science Department \\ Abbassia, Cairo, Egypt \\ \{huss20@hotmail.com,huss@img.cs.titech.ac.jp\}
}

\begin{abstract}
Facial aging simulation and animation are aspiring goals and challenging tasks because the growth and aging processes are greatly influence the facial shape and its structure. The face structure, the size of the bones, and the skin texture change, the skin fattens or sags, wrinkles appear and even muscular activities change in terms of intensity. This paper proposes a novel facial animation algorithm with two techniques integrating such items. These techniques discuss the facial deformation based on the face anthropometry theory and simulates wrinkle with what we call the BRDF (Bidirectional Reflectance Distribution Function) quotient image technique. Given some neutral face $F$, the idea is to capture two characteristics of $F$ with advancing years. The first is the geometric deformation details like skin texture given in $F$ after passing years. The second is the anthropometric data change that developed in the face anthropometry measurements theory. Then, together with warping technique we map these characteristics to any other particular person's face in order to generate more expressive and convincing facial senility. The original contribution and advantages of this paper compared with the other proposed methods are that, the proposed techniques are simple to implement, reliable in which they require only one source image without needing to collect a lot of images and their computations are fast enough for an interactive environment. Experimental results demonstrate our approach with a variety of facial aging animation.
\end{abstract}

\footnotetext{
${ }^{2}$ Visiting Researcher at: Graduate School of Information Science \& Engineering, Tokyo Institute of Technology,

2-12-1 O-okayama, Meguro-ku, Tokyo 152-8552, Japan.
}

\section{Introduction}

One of the most elusive goals in computer animation is the realistic animation of the human face. Human face modeling and animation are very complex tasks because of the physical structure of the face and the dynamics involving its psychological and behavioral aspects. After passing years there are a lot of changes in the face that called facial senility or facial aging. Transforming facial images along perceived dimensions (such as age, gender, race, or health) has wide applications. They include animations, automated face recognitions, educations, entertainments, telecommunications, criminal objects (like finding murders after number of years), finding missing peoples and they used for medical surgery and reconstruction of face [11, 27]. Facial aging is one of the natural phenomena which will happened in the person's face. Observation of the details, such as expressive wrinkles, marks of aging, and conversational signals in the speech, is an important feature of understanding human faces. Wrinkles are important for understanding and interpreting facial expression and also major aspects in determining age. As skin changes with age, wrinkles appear and become permanent and more pronounced. Human faces are distinctively individual, and so are the wrinkles on them. This paper focuses on the simulation of realistic facial aging by concentrating on the medical aspects of the facial deformation and the appearance of wrinkle.

The paper is organized as follows. Section 2, reviews some of the previous work in wrinkle simulation with skin modeling and aging with some discussions of the simulation problems. Section 3, provides a brief summary of the medical face anthropometry theory which we have used. Section 4 , introduces our proposed techniques for facial deformation, wrinkle simulation and aging animation. Some experimental results with discussion are given in section 5 . 
Finally, conclusion and direction for future work are discussed in section 6 .

\section{Previous Work and Problems}

Wrinkle simulation with skin modeling and aging are extremely important in enhancing the realism of human figure models. Varied models are used to simulate skin deformation for different purposes. There are geometric models, physically-based models and biomechanical models using either particle system, or continuous system. Ishii et al. [10] proposed a geometric model of micro wrinkle which uses a curved surface on a based on a polygon for expressing folds and ridges. Other extensions and improvements methods have been reported in $[18,2,20,12]$.

The idea of changing object appearance with only image information has been investigated recently by various researchers in both computer vision and graphics communities. Liu et al. [28] used the image ratio between a neutral face and an expression face of the same person to modify a different person's neutral face image and generate facial expression details. Given a face under two different lighting conditions and another face under the first lighting condition, Shashua et al. [30] used quotient image to generate an image of the second face under the second lighting condition. Stoschek [29] incorporates image morphing into the image synthesis process for photo-realistic re-rendering of faces at arbitrary illumination and pose directions. Another class of techniques based on morphing and their extensions are discussed in $[3,5,9,33]$. There were a few efforts for the dynamic model of wrinkles. Viaud et al. [15] have presented a generic hybrid model for the formation of expressive wrinkles, where bulges are modeled as spline segments.

Although, we have some techniques for wrinkle simulation with skin modeling and aging, model based on the complete description of the head's anatomy doesn't exist because of its complexity as well as the compromise between interactive speed and realism. Texture mapping offers a good simulation of static wrinkles, but constructing visually interesting bump maps requires practices and artistic skills [2]. On the other hand, there is no method in which the texture image modified as a function of skin deformation. A physically based approach [14] can offer a better simulation of wrinkle formation, but if it requires geometrical modeling of the wrinkles it may be expensive. Cloning method and aging simulation in a family [12] generate aging wrinkles on the faces of the son and daughter. Although, this method offered a good simulation of skin aging and wrinkles, its assumption is not satisfied for all families because similarity can not be recognized in all families. Caricaturing technique $[3,5]$ suffers from average face depen- dency and needs a collection of multiple images of different faces to form the average one.

The original contribution of this paper compared with the previous mentioned work is given in terms of the following advantages. First, the proposed techniques are efficient in time complexity, simple to implement, relatively easy, and reliable in which they require only one source image without needing to collect a lot of images. Second, they are local. By local we mean that, wrinkling effects caused by deformations are confined the deformed areas. Third, they provide a simple way to capture the geometrical details founded in images without any constraints and map them smoothly to other images. Finally, their computations are fast enough for an interactive environment.

\section{Face Anthropometry Theory}

In facial animation, computational complexity is a fundamental constraint and real-time performance is often much more important than a highly accurate facial shape in terms of a still image. Up to now, little has been done to simulate the aging process. It is quite cumbersome process which is related to the change of physical structure and biological composition in body tissues. The form and values of these changes can be measured according to face anthropometry, the science dedicated to the measurement of the human face. From this point of view our idea is derived to simulate facial aging process based on face anthropometry $[7,8,17]$. Anthropometry is the biological science of human body measurement. Anthropometric evaluation begins with the identification of particular locations on a subject, called landmark points, defined in terms of visible or palpable feature (skin or bone) on the subject. Farkas [7] used a wide set of measurements for describing the human face. Such measurements consist a total of 47 landmark of point for describing the face. Figure 1, illustrates some of them. The landmarks are typically identified by abbreviations of the corresponding anatomical terms. For example, the inner corner of the eye is "en" for endocanthion, while the top of the flap of cartilage (the tragus) in front of the ear is " $t$ " for tragion. Farkas [7] included some types of facial measurements, which illustrated in figure 2 . In figure 2 , en $-e x$ refers to the shortest distance between the landmarks at the corner of the eye, $v-t r$ refers to the vertical distance between the top of the head and hairline, and $c h-t$ refers to the tangential distance from the corner of the mouth to the tragus. If one asks the question, after passing years, how much variations are there in the face ?, and which feature will be changed with age ?. Medical face anthropometry theory founded the answer and showed us all of the feature which have variations with advancing years. It classified the aging process into four groups, where each group has 
some feature variations with advancing years. More details founded in $[4,7,35,21]$. In this paper, a similar use of anthropometry for facial aging animation will be discussed from the medical point of view with the aid of quotient image concept [30, 29].

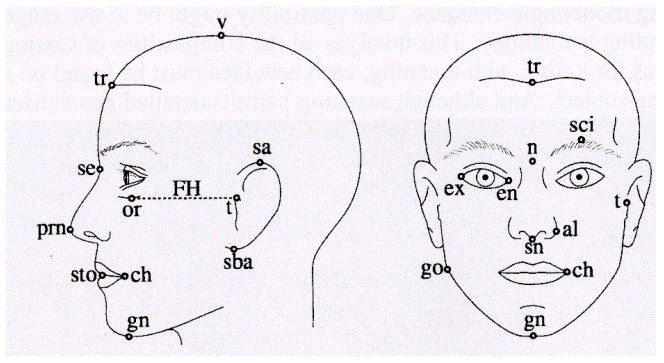

Figure 1. Anthropometric Landmarks on the face $[7,35]$.

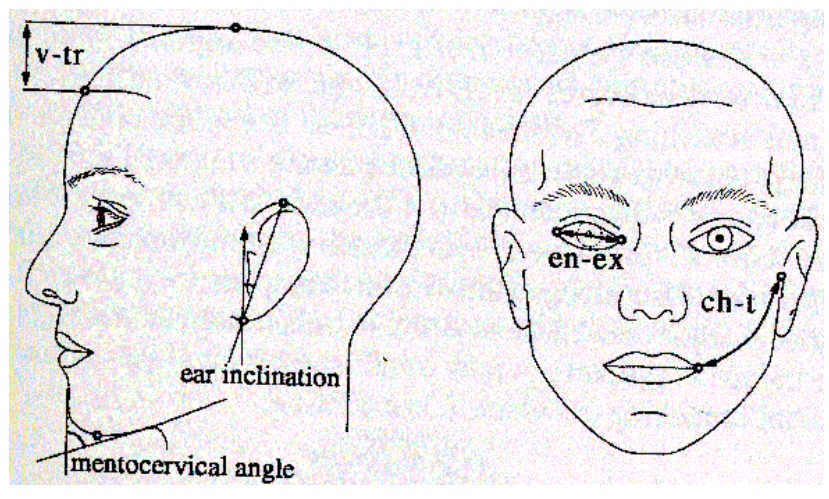

Figure 2. Anthropometric Measurements [7, 35].

\section{The Proposed Techniques}

Face modeling and animation considering wrinkle formation and aging are an aspiring goal and a challenging task. Three main elements have to be considered in facial aging: aging wrinkles, skin texture variation and facial shape change. This section shows how to simulate and predict aging accounting for these three elements. We shall explore a new proposed and an effective approach for facial aging animation and wrinkle simulation. Two proposed techniques will be introduced, one of them is for the face deformation and the other is for the simulation of wrinkle. Our idea is derived from the study of the previously discussed medical face anthropometry theory with the aid of quotient image concept, and $B R D F$ (Bidirectional Reflectance Distribution Function).

\subsection{Facial Deformation Simulation Technique}

As people become older their faces are subject to many changes. For instance, The head undergoes a global remodeling that effects the increased marks in the size of the face relative to cranium. Wrinkles creases, bags, and wattles are developed in response to biomechanical stresses like gravity. Progressive changes in skin texture and hair color, as well as loss of hair (balding) also occur with advancing years. Each of these characteristics can precisely provide perceptual information about the person's age because each is produced by continuous changes resulted from the physical and biomechanical stresses occurring throughout the lifespan $[13,21]$. The idea for the facial deformation technique is based on the way of integrating such items in order to indicate the age of a person with other aging artifacts. The rich description of human geometry that developed in the face anthropometry provides an invaluable resource for human modeling in computer graphics and other applied areas. From face anthropometry medical point of view, the human face can be divided into three balanced regions $[4,7,8,21]$. The first region is from the hairline to the eyebrows, the second region is from the eyebrows to the lower end of the nasal area, and the third region is from the upper lip to the chin. Each region contains some facial landmark points measurements obtained from the previously discussed medical face anthropometry. With advancing years, these balance will change. For example, the hairline will recede, and because of the bending and stretching in the cartilage, nose, ears, other features will have declination too, which in turn affects the overall appearance of the face. Such change will be a function of some threshold which is given in terms of age, sex, weight, and other parameters. For instance, there is some threshold for hair receding [6], or there is some coefficient for cartilage growth with aging $[4,21]$. Animating a familiar object from a single image with aging or under some source of variation requires a handle on how to capture the image space created by that source of variation. Burson et al. [1] computed the difference of the aligned images of a young face and an old face. Given the image of a new neutral face to be aged the difference image is warped and added to this new face to make it look older. Our idea is built on such a technique. The advantage is that, instead of computing such differences, we capture such variations from the study of the previously discussed face anthropometry theory. Therefore, the problem for the facial deformation technique can be formulated mathematically with the aid of the quotient image concept [30] as follows: Given two neutral faces $M_{1}$ and $M_{2}$, such that for every point on $M_{1}$, there is a corresponding point on $M_{2}$ which has the same meaning like, (eye corners, nose tip, mouth corners, etc.). Let $\hat{M}_{1}$ denote the image of $M_{1}$ after deformations due to aging, and $\hat{M}_{2}$ 
be the unknown image of the face $M_{2}$, with the same aging effects (deformations) as $\hat{M}_{1}$. Since human faces have approximately the same geometrical shape, and by assuming that these images have be aligned, then from the concept of the quotient image technique, we have,

$$
\begin{aligned}
\frac{\hat{M}_{1}(x, y)}{M_{1}(x, y)} & =\frac{\hat{M}_{2}(x, y)}{M_{2}(x, y)} \\
& \mapsto \hat{M}_{2}(x, y)=M_{2}(x, y) \frac{\hat{M}_{1}(x, y)}{M_{1}(x, y)}
\end{aligned}
$$

where $(x, y)$ are the coordinates of the pixels in the images, and the ratios $\frac{\hat{M}_{1}(x, y)}{M_{1}(x, y)}$ are known as the quotient image. The idea of the proposed approach is to capture these ratios with aging from the face anthropometric measurements. Anthropometric measurements made a variety of statistical investigations possible on the reliable differences in shape across faces within a group of subjects. Subjects have been grouped on the basis of gender, race, age, attractiveness, etc. Besides the statistics on measurements, statistics on the proportions between measurements have also been derived. These proportions give useful informations about the correlations between features with aging process. Means and variances for the measurements within a group tabulated in $[7,8,21,35]$, effectively provide a set of measurements which virtually capture all of the variations that can be occurred in the group. Taking into account the parameters of the given neutral face $M_{2}$, and its unknown image $\hat{M}_{2}$ after deformations due to aging given in equation (1), the process of our algorithm can be summarized in the following three main steps. The first step is to identify the landmark point measurements or features of the given face, either manually or using some automatic method. The second step is to capture the calculated feature change ratios due to aging, $T=\frac{\hat{M}_{1}(x, y)}{M_{1}(x, y)}$, obtained according to the face anthropometry measurements $[7,8,35]$. The third step is to compute equation (1) by moving the features of $M_{2}$, along $T$, and warp the image accordingly. In our implementation, we used linear warping over the feature points Delaunay triangulation, which we can combine with a one-toone constraint algorithm to prevent the warped image folding $[31,32]$. The process of our algorithm resembles image metamorphosis (morphing) [33], but unlike morphing, which changes the identity of the subject during the morph, by this method the identity is maintained across the transformation as it will be shown in the experimental results.

\subsection{Facial Skin Texture Simulation Technique}

The process of aging depends not only on the structure of the initial face but also on many aspects' of one's life including climatic, psychological and other parameters. The complexity of facial animation is mainly due to the fact that there is no unique model integrating all primary and secondary effects perceived on a face: each particular aspect requires specific modeling and all aspects intersect each other in a complicated manner. Therefore, the previously discussed face deformation technique is not sufficient to give us the accurate impression of the aging process. The appearance of wrinkle due to facial expressions and aging adds realism to the modeling and animation especially with advancing years. This section focuses on the simulation of facial texture such as stubble and wrinkles. The idea is derived from the previously discussed face anthropometry theory $[7,8,17]$, the geometry of the surface bidirectional reflectance distribution function (BRDF) [20, 22], and the quotient image concept [30] of two coinciding objects. Two objects $T_{1}$ and $T_{2}$ are said to be coincident, if there exists a continuous function $G$ from $T_{1}$ into $T_{2}$, such that if $\left\|x_{1}-x_{2}\right\| \leq \beta$, for $x_{1} \in T_{1}$, and $G\left(x_{1}\right)=x_{2} \in T_{2}$, then their average computed normal $\hat{N}$ defined relative to their neighborhoods $\chi\left(x_{1}\right)$, and $\chi\left(x_{2}\right)$ respectively satisfy the following condition: $\left\|\hat{N}\left(x_{1}\right)-\hat{N}\left(x_{2}\right)\right\| \leq \gamma$, where $\gamma$, and $\beta$ are two small positive numbers. The technique is based on the observation that wrinkle appearance from any of the three regions of the balance face previously discussed in section 4.1 can be captured from a single image of an object in a way independent of its surface reflectance. Then, using the concept of quotient image given in [30], this wrinkle is mapped to the appearance of other face to make it look older. In the following we will summarize the fundamental definitions and basic notions of BRDF, quotient image concept and finally introduce our proposed technique.

\subsubsection{Bidirectional Reflectance Distribution Function (BRDF) Overview}

The bidirectional reflectance distribution function (BRDF) of a surface describes how light is scattered at its surface $[23,20]$. It completely describes the reflectance of a surface at a single point. Its value measures the ratio of the radiance $L$ exiting the surface in a given direction to the incident irradiance $I$ at a particular wavelength $\lambda$ from an incident solid angle $d \omega_{i}$ about a given illumination direction $[19,20,22]$. The (BRDF) denoted by $f_{r}$, is defined according to figure 3 , as follows:

$$
f_{r}\left(\theta_{i}, \phi_{i}, \theta_{e}, \phi_{e}, \lambda\right)=\frac{L_{r}\left(\theta_{e}, \phi_{e}\right)}{L_{i}\left(\theta_{i}, \phi_{i}\right) \cos \theta_{i} d \omega_{i}}
$$

where, $L_{r}=L_{r, s}\left(\theta_{e}, \phi_{e}\right)+L_{r, v}\left(\theta_{e}, \phi_{e}\right)$ is the reflected radiance which has two components, one is the reflected radiance due to surface scattering $L_{r, s}$, and the other component $L_{r, v}$ is due to the subsurface volume scattering. The (BRDF) is thus a function of five variables, but its domain is reduced somewhat by a symmetry called reciprocity. Reciprocity stated that reversing 


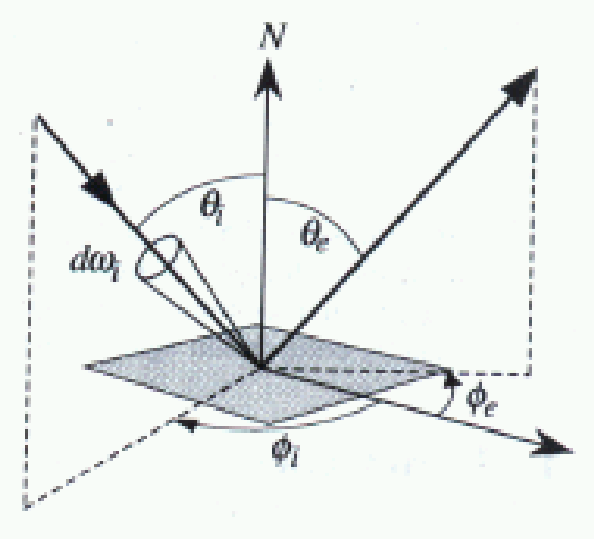

Figure 3. Geometry of surface reflection with normal vector $N$.

the lights path does not change the reflectance, that is, $f_{r}\left(\theta_{1}, \phi_{1}, \theta_{2}, \phi_{2}, \lambda\right)=f_{r}\left(\theta_{2}, \phi_{2}, \theta_{1}, \phi_{1}, \lambda\right)$.

\subsubsection{BRDF Quotient Image}

Given two coinciding image objects $A$ and $B$, the quotient image $Q$, is defined by the ratio of their albedo functions $\frac{\rho_{A}}{\rho_{B}}$ [19]. Formally, the quotient image $Q_{A}$ of object $A$ against object $B$, is defined by,

$$
Q_{A}(x, y)=\frac{\rho_{A}(x, y)}{\rho_{B}(x, y)},
$$

where $x$ and $y$ are the range over the image. So, the image $Q_{A}$ depends only on the relative surface texture information and thus, is independent of illumination. This will be clear from the following proposition which is proved in [30].

[Proposition 1] Given three images $\left\{a_{1}, a_{2}, a_{3}\right\}$ of object $\{a\}$ illuminated by three linearly independent lighting conditions, and an image $\left\{y_{s}\right\}$ of object $\{y\}$ illuminated by some light source $(s)$, then there exists coefficients $\left\{x_{1}, x_{2}, x_{3}\right\}$, that satisfy,

$$
y_{s}=\sum_{j} x_{j} a_{j} \oplus Q_{y},
$$

where $\oplus$ denotes the Cartesian product (pixel by pixel multiplication). Moreover, the image space of object $y$ is spanned by varying the coefficients.

Now, for a given two coinciding images $A$ and $B$, equation (2), can be rewritten relative to both $A$ and $B$ as follows:

$$
f_{r_{A}}\left(\theta_{i}, \phi_{i}, \theta_{e}, \phi_{e}, \lambda\right)=\frac{L_{r_{A}}\left(\theta_{e}, \phi_{e}\right)}{L_{i_{A}}\left(\theta_{i}, \phi_{i}\right) \cos \theta_{i_{A}} d \omega_{i_{B}}},
$$

and

$$
f_{r_{B}}\left(\theta_{i}, \phi_{i}, \theta_{e}, \phi_{e}, \lambda\right)=\frac{L_{r_{B}}\left(\theta_{e}, \phi_{e}\right)}{L_{i_{B}}\left(\theta_{i}, \phi_{i}\right) \cos \theta_{i_{B}} d \omega_{i_{B}}} .
$$

Since human faces have approximately the same geometrical shape and since the two images are coincident, then using the concept of the quotient image given by equation (3), we have, $Q_{A}=\frac{f_{r_{A}}}{f_{r_{B}}} \cong \frac{\cos \theta_{i_{B}}}{\cos \theta_{i_{A}}}$. Intuitively, the BRDF represents for each incoming angle, the amount of light that is scattered in each outgoing angle, where cosine can be written as a dot product of normalized versions of the light source vector and the normal vector. This relationship between brightness and surface orientation is often called Lambert's law [26, 22]. For a Lambertian (perfectly diffuse) surface, the BRDF is constant, and equal to $\frac{\rho_{d}}{\pi}$, where, $\rho_{d}$ is the diffuse reflection coefficient, and the factor of $\pi$ is necessary so that the BRDF is correctly normalized. From this point of view, and since geometrically, the difference between an old person's skin surface and a young person's skin surface is that, the older one has more bumps than the younger one, then for a diffused surface, the problem of facial skin texture (such as wrinkle) simulation can be stated as follows: Given images $M_{1}$ and $M_{2}$ of two coinciding surfaces $C_{1}$ and $C_{2}$ respectively, what is the new form of the image $\hat{M}_{2}$ of $C_{2}$, if we modify its surface normal $\hat{N}_{2}$ such that, for two corresponding points $p_{1} \in C_{1}$ and $p_{2} \in C_{2}$, we have,

$$
\hat{N}_{2}\left(p_{2}\right)=N_{1}\left(p_{1}\right)
$$

\subsection{Wrinkle Simulation Re-Rendering Technique}

In order to solve equation (5), we will capture the facial skin texture using the quotient image concept (Equ. (3)) as follows: For any point $p$ on the diffused surface $C$, let $\rho_{d}$ be its diffusion reflection coefficient, $N$ be its normal vector at $p, \ell$ denote the light direction from $p$ to the light source, $I_{s}$ be its intensity (extension to multiple light sources is straight forward), and $v$ be the direction vector from $p$ to the viewers. Then, according to the Lambertian model, the intensity of the point $p$ in the image $M$ is given as follows:

$$
M(p)=I_{s}(p) N(p) \rho_{d}(p) \ell
$$

As discussed before, since both surfaces of the two images are coincident, (i.e. $\left\|p_{1}-p_{2}\right\| \leq \beta$, where $\beta$ is a small positive number), this leads to the approximation $I_{s}\left(p_{2}\right) \cong I_{s}\left(p_{1}\right)$. Then from equations (3), (5), and (6), we have:

$$
\frac{\hat{M}_{2}\left(p_{2}\right)}{M_{2}\left(p_{2}\right)}=\frac{I_{s}\left(p_{2}\right) \hat{N}_{2}\left(p_{2}\right) \rho_{d}\left(p_{2}\right) \ell}{I_{s}\left(p_{2}\right) N_{2}\left(p_{2} \rho_{d}\left(p_{2}\right)\right) \ell} \cong \frac{I_{s}\left(p_{1}\right) N_{1}\left(p_{1}\right) \rho_{d}\left(p_{2}\right) \ell}{I_{s}\left(p_{2}\right) N_{2}\left(p_{2}\right) \rho_{d}\left(p_{2}\right) \ell},
$$

multiply by $\frac{\rho_{d}\left(p_{1}\right)}{\rho_{d}\left(p_{1}\right)}$, and use equation (6), we get,

$$
\frac{\hat{M}_{2}\left(p_{2}\right)}{M_{2}\left(p_{2}\right)} \simeq \frac{M_{1}\left(p_{1}\right) \rho_{d}\left(p_{2}\right)}{M_{2}\left(p_{2}\right) \rho_{d}\left(p_{1}\right)} \mapsto \hat{M}_{2}\left(p_{2}\right) \simeq \frac{M_{1}\left(p_{1}\right) \rho_{d}\left(p_{2}\right)}{\rho_{d}\left(p_{1}\right)}
$$


From equation (7), the quotient image between two images is the ratio of their albedo (texture) functions $\frac{\rho_{d}\left(p_{2}\right)}{\rho_{d}\left(p_{1}\right)}$. Computer visions techniques are very useful for modeling real world objects as well their geometric details. Zheng and Chellappa [19] concluded that, in computer vision shape from shading is implemented by first modeling imaging model as a function of the surface geometry. Then surface is reconstructed and under the given imaging model, shaded image is generated close to the input. Therefore, in order to calculate $\frac{\rho_{d}\left(p_{2}\right)}{\rho_{d}\left(p_{1}\right)}$, we define the smoothed image of $M$ as follows:

$$
\tilde{M}(p) \cong \sum_{\chi(p)} \kappa(\chi(p)) M(\chi(p))
$$

where, $\chi(p)$ is the neighborhood of $p, \kappa(\chi(p))$ is some kernel function of a smooth filter [34], say, a mean filter, median filter, or Gaussian filter of the neighborhood of $p$, and $M(\chi(p))$ is the recorded intensity in the neighborhood $\chi(p)$ of a point $p$ in the image $M$. Let the size of $\chi(p)$ be relatively small in compared with its distance to the light source then, $I_{s}(p) \simeq(\chi(\quad \quad p))$. Assume also that $\rho_{d}(p) \simeq$ $\rho_{d}(\chi(p))$. Thus, from equations (8) and (6), we have,

$$
\tilde{M}(p)=\rho_{d}(\chi(p)) I{ }_{s}(\chi(p)) \hat{N}(p) \cong \rho_{d}(p) \ell I_{s}(p) \hat{N}(p),
$$

where, $\hat{N}(p)=\sum_{\chi(p)} \kappa(\chi(p)) N(\chi(p))$ is the average normal relative to $\chi(p)$. Now, given images $M_{1}$ and $M_{2}$ of two coincident surfaces $C_{1}$ and $C_{2}$ respectively, we get from equations (3) and (9), that,

$$
\frac{\tilde{M}_{2}\left(p_{2}\right)}{\tilde{M}_{1}\left(p_{1}\right)} \simeq \frac{\rho_{d}\left(p_{2}\right) I_{s}\left(p_{2}\right) \hat{N}\left(p_{2}\right) \ell}{\rho_{d}\left(p_{1}\right) I_{s}\left(p_{1}\right) \hat{N}\left(p_{1}\right) \ell}
$$

As mentioned before, since both $M_{1}$ and $M_{2}$, are coincident, then, $\left\{I_{s}\left(p_{1}\right) \approx I_{s}\left(p_{2}\right)\right\}$, and $\left\{\hat{N}\left(p_{1}\right) \approx \hat{N}\left(p_{2}\right)\right\}$. So, equation (10) can be rewritten as,

$$
\frac{\tilde{M}_{2}\left(p_{2}\right)}{\left.\tilde{M}_{(1} p_{1}\right)} \simeq \frac{\rho_{d}\left(p_{2}\right)}{\rho_{d}\left(p_{1}\right)}
$$

Substitute from equation (11) into (7), we get:

$$
\left.\left.\hat{M}_{2} p_{2}\right) \simeq \frac{\tilde{M}_{2}\left(p_{2}\right)}{\tilde{M}_{1}\left(p_{1}\right)} M_{(1} p_{1}\right)
$$

Based on the $B R D F$ with quotient image concept, equation (12) represents the solution of equation (5). It shows, how one can capture the image space created by some source of variation from a single image with aging process. It also shows that a smooth in the image space domain is correspond to a smooth in the geometrical space domain when the surface reflectance is smooth. The process of the proposed algorithm for two given images $M_{1}$ and $M_{2}$ of coinciding shapes can be summarized in three main steps.
The first step is the identification of the landmark points measurements or features of the given faces. The forms and values of these measurements are computed according to the face anthropometry theory $[7,8,17]$. The second step is the pixel alignment through image warping [16]. In our implementation, we used linear warping over the feature points Delaunay triangulation, which we can combine with a one-to-one constraint algorithm to prevent the warped image folding [31, 32]. Once the alignment is done, the final step is to compute equation (12) via some smoothing filter. In such a step, any smoothing filters can be used, in the implementation, we used the Gaussian as a smoothing filter due to its frequency response [34]. The effectiveness of the Gaussian function is different for different choosing the standard deviation sigma of the Gaussian filter. The process of our algorithm yields images with realistic looking wrinkle simulation without needing to model each wrinkle as a separate element.

\section{Experimental Results and Discussion}

Several simulations for facial aging animations have been performed based on our proposed techniques. Figures 4 and 5 show two facial aging animations using the facial deformation technique. Figure 6, demonstrates more expressive and convincing facial aging animations with different sigma value of the Gaussian filter by combining the facial deformation and the wrinkle simulation techniques. From the experimental results, one can see that in compared with image metamorphosis (morphing) [33], the neutral face identity is maintained during the animation as discussed in the last paragraph of section 4.1.

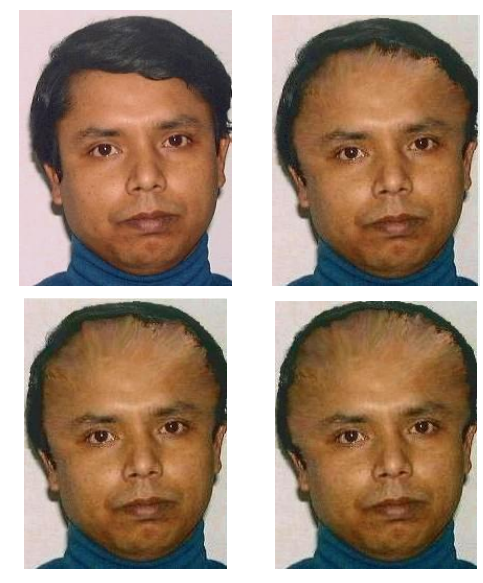

Figure 4. Facial aging animation results by facial deformation technique. 

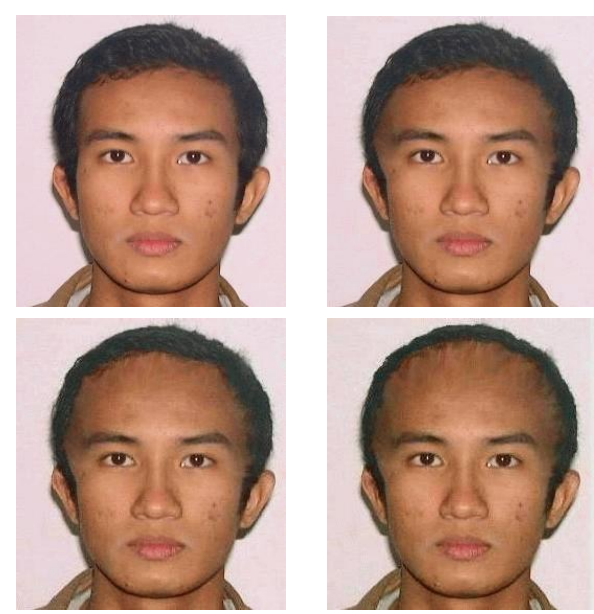

Figure 5. Another Facial aging animation results by facial deformation technique.

\section{Conclusion and Future Work}

Modeling and rendering of wrinkle simulation with human skin aging animation are difficult tasks and challenging problems. Many different approaches have been proposed for facial aging animation. This paper presented a new and effective image modeling techniques for such an animation based on the medical face anthropometry theory and the concept of quotient image technique. Two proposed techniques have been introduced, one for the facial deformation, and the other for the wrinkle simulation. The advantages of the proposed techniques over the previous proposed methods are given as follows. First, the proposed techniques are efficient in time complexity, simple to implement, and reliable in which they require only one source image without a need to collect a lot of images. Second, they are local. By local we mean that, wrinkling effects caused by deformations are confined the deformed areas. Third, they provide a simple way to capture the geometrical details founded in images without any constraints and map them smoothly to other images. Finally, their computations are fast enough for an interactive environment. On the other hand, the process of aging is the complex one. It depends not only on the structure of the initial face but also on many aspects of one's life, including climatic, psychological and other parameters. Consequently, for more realistic visual aging effects, further research effort is still needed. Our future work will focus on two categories. First, we would like to extend our algorithm based on spherical Wavelets [25] by using what they refer to as a lifting scheme. Second, investigating the work of [24], and extend our algorithm for facial aging animation using fractal theory. Fractal theory has the property of self-similarity which is similar to the aging process.

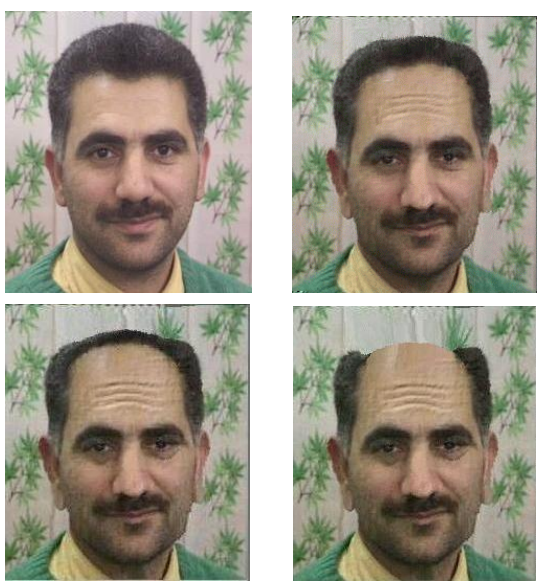

Figure 6. Facial aging animation results by combining wrinkle simulation and facial deformation techniques.

\section{References}

[1] N. Burson and D. Schneider, "Methods and apparatus for producing an image of a person's face at a different age”, United States Patent 4276570, p. 1-14, 1981.

[2] L. Boissieux, G. kiss, N. Magnenant Thalman and P. Kalar, "Simulation of Skin Aging and Wrinkle with Cosmetic Insight", Computer Animation and Simulation, pp.15-27, 2000.

[3] D. Burt and D. Perrett, "Perception of age in adult Caucasian male faces: computer graphic manipulation of Shape and color information”, Proc. Royal Soc. Lond. B 259, pp.137-143, 1995.

[4] J. Cheng, M. Stephen, W.Perkins and M.Hamilton, "Perioral Rejuvenation", Proc. of Facial Plastic Surgery Clinics Of North America, Vol.8, No. 2, pp.223, MAY 2000.

[5] A. Duncan and I. David, "Manipulating Facial Appearance through shape and color", IEEE Computer Graphic and Applications, Vol. 15, No. 5, pp. 70-76, 1995.

[6] T. Ezaki and Y. Kasori, "Bilateral Temporoparietal Flaps in the Treatment of Male Baldness", Aesth. Plast. Surg., Vol. 19, pp. 41-47, 1995.

[7] L. Farkas, "Anthropometry of the Head and Face", Raven Press, 1994.

[8] L. Farkas,"Anthropometric facial proportions in medicine", Thomas Books, 1987. 
[9] A. George, G.H.Hole, "The influence of Feature based information in the age processing of unfamiliar face", Perception, Vol.27, pp.295-312, 1998.

[10] T. Ishii, T. Yasuda, S. Yokoi and J. Toriwaki, "A Generation Model for human skin texture", Proc. of CGI'93, pp. 139-150,1993.

[11] M. Koch, M. Gross, F. Carls , D. Buren, G. Fankhauser, Y. Parish, "Simulation facial surgery using finite element models", Proc. of SIGGRAPH'96, pp. 421-428, 1996.

[12] W. Lee, Y. Wu, N. Magnenat-Thalmann, "Cloning and Aging in a VR family", The Visual Computer, Vol. 5, No. 1, pp. 32-39, 1999.

[13] S. Mark, J. Pittenger, and H. Hines, "Wrinkle and head shape as coordinated source of age-level information ", Perception \& Psychophysics, Vol. 27, No. 2, pp. 117-124,1998.

[14] D. Terzopoulos and K. Waters "Physically-Based facial Modeling And Animation ", Journal of Visualization and Computer Animation, Vol. 1, pp. 73-80, 1990.

[15] M. Viaud, and H. Yahia,"Facial animation with Wrinkles", $3^{\text {rd }}$ Workshop on animation, Eurographics'92, Cambridge, Springer-Verlag, 1992.

[16] G. Wolberg, "Digital Image Warping", IEEE Computer Society Press, 1990.

[17] D. Douglas, D. Metaxas, M. Stone, "An Anthropometric face model using Variational Techniques", Proc. of SIGGRAPH'98, pp. 67-74, 1998.

[18] Y. Wu, L. Moccozet and N. Thalman, "Simulation of Wrinkle and Skin Aging", Visualization and Computer Animation, Vol. 15, pp. 183-195, 1999.

[19] Q. zheng and R. Chellappa, "Estimation of illumination direction, albedo, and shape from shading", IEEE Transaction PAMI, 13(7), pp. 680-702, 1991.

[20] R. Marschner, H. Westin, P. Lafortune, E. Torrance, and P. Donald, "Image-based BRDF measurement including human skin". In Eurographics Workshop on Rendering, 1999.

[21] H. Kwon and N. Lobo, "Age classification from facial images", Computer Vision and Image Understanding, Vol. 74, No. 1, pp. 1-21, 1999.

[22] D. Xiao, E. Torrance, X. Sillion and P. Greenberg, "A comprehensive physical model for light reflection ", SIGGRAPH'91, pp. 175 - 186, 1991.
[23] P. Hanrahan, and W. Kruegar, "Reflection from layered surfaces due to subsurface scattering", SIGGRAPH'93, pp. 165-174, 1993.

[24] J. Kautz, W. Heidrich and H. Seidel, "Real-Time Bump Map Synthesis", Proceedings of the Eurographics/SIGGRAPH Workshop on Graphics Hardware 2001, pp. 109-114, August 2001.

[25] S. Peter, and S. Wim, "Spherical Wavelets: efficiently representing functions on the sphere", Proc. of SIGGRAPH'95, pp. 161-172, 1995.

[26] M. Oren and K. Nayar, "Generalization of Lambert's reflectance model”, SIGGRAPH'94, pp. 239 - 246, 1994.

[27] R. Marschner, B. Guenter and S. Raghupathy. "Modeling and Rendering for Realistic Facial Animation", In Proceedings of $11^{t} h$ Eurographics Workshop on Rendering. Held in Brno, Czech Republic, June 2000.

[28] Z. Liu, Y. Shan and Z. Zhang, "Expressive expression mapping with ratio images", Proc. of SIGGRAPH'2001, pp. 271-276, 2001.

[29] A. Stoschek, "Image-based re-rendering of faces for continuous pose and illumination directions", IEEE Conf. on CVPR, pp. 582-587, 2000.

[30] A. Shashua and R. Tammy, "The Quotient Image: Class-based re-rendering and recognition with varying illuminations", IEEE Transaction PAMI, 23(2), pp. 129-139, 2001.

[31] B. Tiddeman, N. Duffy and G. Rabey, "A general method for overlap control in image warping", Computers and Graphics, Vol. 25, No. 1, pp. 59-66, 2001.

[32] F. Kikuo and M. Mihail, "Foldover-free image warping", Graphical Models and Image Processing, Vol. 60, No. 2, pp. 100-111, 1998.

[33] H. Karam, A. Hassanien and M. Nakajima, "Feature Based Image Metamorphosis Optimization Algorithm", IEEE International Conference on Virtual Systems and Multimedia, VSMM'2001, pp. 555- 564, USA, 2001.

[34] R. Gonzalez and R. Woods, Digital Image Processing. Addison-Wesley Publishing Company, 1992.

[35] J. Kolar and E. Salter, Craniofacial Anthropometry: Practical measurements of the head and face for clini$\mathrm{cal}$, surgical and research use. Charles C. Thomas Publisher, LTD, 1996. 